\title{
Comparison of lung cancer cell lines representing four histopathological subtypes with gene expression profiling using quantitative real-time PCR
}

Takashi Watanabe ${ }^{1}$, Tomohiro Miura', Yusuke Degawa', Yuna Fujita ${ }^{1}$, Masaaki Inoue $^{2}$, Makoto Kawaguchi ${ }^{3}$, Chie Furihata ${ }^{{ }^{*}}$

\begin{abstract}
Background: Lung cancers are the most common type of human malignancy and are intractable. Lung cancers are generally classified into four histopathological subtypes: adenocarcinoma (AD), squamous cell carcinoma (SQ), large cell carcinoma (LC), and small cell carcinoma (SC). Molecular biological characterization of these subtypes has been performed mainly using DNA microarrays. In this study, we compared the gene expression profiles of these four subtypes using twelve human lung cancer cell lines and the more reliable quantitative real-time PCR (qPCR).

Results: We selected 100 genes from public DNA microarray data and examined them by DNA microarray analysis in eight test cell lines (A549, ABC-1, EBC-1, LK-2, LU65, LU99, STC 1, RERF-LC-MA) and a normal control lung cell line (MRC-9). From this, we extracted 19 candidate genes. We quantified the expression of the 19 genes and a housekeeping gene, GAPDH, with qPCR, using the same eight cell lines plus four additional validation lung cancer cell lines (RERF-LC-MS, LC-1/sq, 86-2, and MS-1-L). Finally, we characterized the four subtypes of lung cancer cell lines using principal component analysis (PCA) of gene expression profiling for 12 of the 19 genes (AMY2A, CDH1, FOXG1, IGSF3, ISL1, MALL, PLAU, RAB25, S100P, SLCO4A1, STMN1, and TGM2). The combined PCA and gene pathway analyses suggested that these genes were related to cell adhesion, growth, and invasion. S100P in AD cells and $\mathrm{CDH1}$ in $\mathrm{AD}$ and $\mathrm{SQ}$ cells were identified as candidate markers of these lung cancer subtypes based on their upregulation and the results of PCA analysis. Immunohistochemistry for S100P and RAB25 was closely correlated to gene expression.
\end{abstract}

Conclusions: These results show that the four subtypes, represented by 12 lung cancer cell lines, were well characterized using GPCR and PCA for the 12 genes examined. Certain genes, in particular S100P and CDH1, may be especially important for distinguishing the different subtypes. Our results confirm that qPCR and PCA analysis provide a useful tool for characterizing cancer cell subtypes, and we discuss the possible clinical applications of this approach.

\section{Background}

Lung cancer is the leading cause of cancer-related death in men and women worldwide and continues to increase in frequency. Currently, a diagnosis of lung cancer is generally based on histopathological findings. Lung cancers are generally classified as either small-cell lung

\footnotetext{
*Correspondence: chiefurihata@gmail.com

${ }^{1}$ Department of Chemistry and Biological Science, School of Science and Engineering, Aoyama Gakuin University, Kanagawa 229-8558, Japan
}

carcinoma (SC) or non-small-cell lung carcinoma (NSCLC). NSCLC is further classified into three histopathological subtypes: adenocarcinoma (AD), squamous cell carcinoma (SQ), and large cell carcinoma (LC). However, progression, metastatic susceptibility, therapeutic and radiation therapy sensitivity, and prognosis cannot be fully predicted based on initial histopathological observations. Molecular characterization of tumors, by assaying gene expression using techniques such as DNA microarray analysis, has the potential to 
significantly inform medical care that is otherwise based on surgical pathology and oncology. Using this technology, it may be possible to identify clinically important subsets of tumors that would otherwise be indistinguishable by conventional histopathological assessment. In principle, expression profiling should identify tumors that are more likely to invade, relapse, and metastasize, and the approach should allow improved prediction of responses to specific therapeutic regimens and clinical outcomes [1-3]. However, recent publications have raised concerns about the reliability of microarray technology for analyzing differential expression, because of the lack of reproducibility across laboratories and platforms despite the use of highly similar protocols [4]. Initial investigations (e.g., 2000-2003) highlighted discrepancies in gene expression analyzed with different microarray technologies [5]. Although a considerable number of studies have used DNA microarrays to genetically identify lung cancer patients and lung cancer cells [1-3,6-10], marker gene candidates have varied depending on the report.

Quantitative real-time PCR (qPCR) is generally considered the "gold-standard" assay for measuring gene expression and is often used to confirm microarray data [11]. qPCR is the most sensitive technique for detection and quantification of mRNA targets [12]. Recently, it has been suggested that qPCR may be a simpler, more reliable, and more reproducible method than DNA microarrays [13]. qPCR has been used as a supplementary technique for characterizing lung cancer cells [14]. The recent development of DNA databases and bioinformatics techniques has made it possible to determine gene pathways and gene networks [15]. Statistical analyses, such as principal component analysis (PCA), have recently proven useful in this field. Establishing molecular profiles of the four histopathological subtypes of lung cancer cells in relation to gene networks and statistical analysis would be a valuable and meaningful undertaking. Because the analysis of DNA microarrays is expensive and complex, it is often not practical for routine diagnosis to use high-throughput DNA microarrays containing more than 10,000 genes. A diagnostic approach designed for less than 100 marker genes using either a smaller, less-expensive DNA microarray or qPCR would be more practical. To classify the four histopathological subtypes, we selected 100 candidate marker genes that showed relatively consistent differential expression in reports that analyzed a total of 580 clinical lung cancer tissues and 64 lung cancer cell lines [1-3,6-10]. We first selected candidate genes using DNA microarrays and then quantified their expression by qPCR. Although clinical application is the ultimate goal, there are some issues to consider when examining clinical tissues with DNA microarrays or qPCR. First, tissues contain varying amounts of contamination from neighboring stromal cells. Second, RNA amplification is required if the amount of clinical tissue is limited, for instance when samples are obtained by microdissection of cancer cells. While these issues are not problematic for analyzing lung cancer cell lines, they become significant barriers when analyzing clinical samples. Finally, the use of epithelial tissue from sites adjacent to tumors as the normal control has drawn criticism [16], as this tissue often includes histologically normal but genetically abnormal cells [17].

In this study, we first selected 100 genes from published studies and used DNA microarrays to examine their expression in eight test cell lines (A549 [AD], ABC-1 [AD], EBC-1 [SQ], LK-2 [SQ], LU65 [LC], LU99 [LC], STC 1 [SC], RERF-LC-MA [SC]) representing four histopathological subtypes of lung cancer cells plus a normal control lung cell line (MRC-9). From this, we identified 19 candidate genes for subtype-specific markers. Second, we quantified the expression of these 19 genes in the different cell lines using qPCR. Third, we evaluated the 19 genes with an additional four validation lung cancer cell lines (RERF-LC-MS [AD], LC-1/sq [SQ], 86-2 [LC], and MS-1-L [SC]) and MRC-9 cell by qPCR. Fourth, we analyzed the data using statistical, bioinformatics, PCA, and gene pathway analysis (Ingenuity Pathways Analysis, IPA). We selected 12 optimal marker genes and demonstrated that these profiles could discriminate the four histopathological subtypes of tumors. In addition, we confirmed the results using immunohistochemical analysis.

\section{Results}

Identification of candidate genes by microarray analysis

We selected 100 genes from public DNA microarray data $[1-3,6-10]$ and examined their expression in eight lung cancer cell lines (A549 [AD], ABC-1 [AD], EBC-1 [SQ], LK-2 [SQ], LU65 [LC], LU99 [LC], STC 1 [SC], RERF-LC-MA [SC]) and a normal control (MRC-9) by DNA microarray analysis. After eliminating low-expressing genes, we calculated the expression ratio of each gene in each cancer cell line relative to the normal cell line. From this, we identified 18 differentially expressed candidate genes based on the results of a Dunnett's test (Table 1). Another one gene, ISL1, was added as a tentative candidate gene because it had more than 10-foldhigher expression in one cell line than any other line (Table 1). The microarray results were deposited in the CIBEX microarray database (accession CBX 100).

\section{Quantification of 19 candidate genes by qPCR}

Using qPCR, we quantified the expression of the 19 candidate genes in the same eight test cell lines (A549, ABC-1, EBC-1, LK-2, LU65, LU99, STC 1, RERF-LCMA) and the normal cell line (MRC-9) (Figure. 1). This 
Table 1 Selection of candidate genes by DNA microarray

\begin{tabular}{|c|c|c|c|c|c|c|c|c|}
\hline \multirow[t]{3}{*}{ Gene } & \multicolumn{8}{|c|}{ Cell lines by histopathological subtype } \\
\hline & \multicolumn{2}{|c|}{$A D$} & \multicolumn{2}{|c|}{ SQ } & \multicolumn{2}{|c|}{ LC } & \multicolumn{2}{|c|}{ SC } \\
\hline & A549 & $A B C-1$ & EBC-1 & LK-2 & LU65 & LU99 & STC 1 & RERF-LC-MA \\
\hline AMY2A & -1.22 & 0.87 & $3.68 * *$ & 0.68 & $2.50 *$ & 1.90 & $2.91 *$ & 1.36 \\
\hline BEX1 & 3.25 & 5.69 & $7.36^{* *}$ & 3.08 & -0.17 & 2.86 & $4.72 *$ & 5.72 \\
\hline$\overline{\mathrm{CDH} 1}$ & 1.85 & $3.70 * *$ & 1.87 & $3.85 * *$ & -0.41 & 5.20 & 0.48 & -0.70 \\
\hline CSTA & $2.54 * *$ & $4.79 * *$ & -1.06 & 0.80 & 0.09 & -1.93 & $2.18 * *$ & 0.33 \\
\hline DUSP4 & $4.24 * *$ & $2.34^{* *}$ & $4.91 * *$ & 1.26 & $4.12 * *$ & 0.96 & -3.21 & $2.16 *$ \\
\hline FOSL1 & -1.26 & -1.73 & $3.27 * *$ & -2.62 & $2.10 * *$ & $2.44 * *$ & -4.13 & $2.17 * *$ \\
\hline FOXG1 & -0.19 & 3.78 & -0.20 & 0.65 & -0.16 & 1.14 & $5.63 * *$ & 0.17 \\
\hline HMGA1 & -0.59 & 0.24 & $2.82 * *$ & 0.82 & $1.68 * *$ & $3.24 * *$ & 0.49 & $2.81 * *$ \\
\hline IGSF3 & $5.61 * *$ & 4.86 & -0.12 & $6.35 *$ & 0.19 & 1.28 & 4.71 & 4.62 \\
\hline INADL & 2.44 & 3.70 & 2.50 & $7.65 * *$ & 0.48 & 1.48 & 0.80 & 2.30 \\
\hline ISL1 & 0.31 & -0.45 & 0.08 & 2.21 & 4.61 & 0.10 & -0.74 & 3.96 \\
\hline MALL & $2.20 * *$ & $2.39 * *$ & $2.44 * *$ & -0.07 & $3.86 * *$ & 1.54 * & N.D. & $2.36 * *$ \\
\hline PLAU & $-3.61 * *$ & $-7.21 * *$ & -0.02 & $-6.75 * *$ & $-1.82 * *$ & $-1.17 * *$ & $-6.95 * *$ & $-1.62 * *$ \\
\hline RAB25 & -0.39 & $6.39 * *$ & 3.37 & $5.33^{* *}$ & 0.65 & 0.44 & -2.87 & -1.18 \\
\hline S100A2 & -0.70 & -0.02 & $4.73 * *$ & 0.08 & $2.95 * *$ & 1.66 & -1.01 & $4.76 * *$ \\
\hline S100P & $6.28 * *$ & $8.57^{* *}$ & -0.47 & 5.00 & $6.80 * *$ & 0.38 & 0.39 & 0.13 \\
\hline SLCO4A1 & 0.13 & 2.92 & 2.81 & 1.52 & $4.74 *$ & $6.67 * *$ & 2.83 & 1.78 \\
\hline STMN1 & -1.44 & $1.41^{* *}$ & $1.49 * *$ & $1.41 * *$ & 0.44 & 0.68 & $2.07 * *$ & $2.21 * *$ \\
\hline TGM2 & -0.56 & $-2.93 * *$ & 0.46 & $-5.61 * *$ & -0.39 & -1.99 & -7.62 ** & $-5.52 * *$ \\
\hline
\end{tabular}

Total RNA was extracted from each cultured cell line and reverse-transcribed to produce cDNA. cDNA samples from cancer cells (labeled with Alexa 555) and from normal cells (labeled with Alexa 647) were mixed and hybridized to a DNA microarray that was then scanned with a DNA microarray scanner ( $\mathrm{n}=6$ ). The ratio of cancer cells to control cells, based on the relative intensities of the two fluorescence signals, was calculated using ArrayGauge. ${ }^{*} \mathrm{p}<0.05$, ${ }^{* *} \mathrm{p}<0.01$ with Dunnett's test.

gave a total of 152 data points $(19 \times 8)$ from each of the DNA microarray and qPCR analyses. Increases or decreases in 118 data points from qPCR were consistent with those from the DNA microarray analysis. Furthermore, qPCR provided more sensitive data than the DNA microarray analysis (Table 1 and Figure. 1). Using qPCR, two AD cell lines showed consistent and significant upregulation in 12 genes $(A M Y 2 A, B E X 1, C D H 1$, CSTA, DUSP4, FOXG1, IGSF3, INADL, ISL1, MALL, S100P, and SLCO4A1) and downregulation in PLAU. Two SQ cell lines showed upregulation in 11 genes (AMY2A, BEX1, CDH1, DUSP4, HMGA1, IGSF3, INADL, ISL1, MALL, RAB25, and SLCO4A1) and downregulation in $P L A U$. Two LC cell lines showed upregulation in 12 genes $(A M Y 2 A, C D H 1, D U S P 4, F O S L 1$, FOXG1, HMGA1, IGSF3, ISL1, MALL, RAB25, S100A2, and SLCO4A1) and downregulated in CSTA. Two SC cell lines showed upregulation in nine genes $(A M Y 2 A$, BEX1, FOXG1, IGSF3, INADL, ISL1, RAB25, SLCO4A1, and STMN1) and downregulation in TGM2 (Figure 1).

\section{Evaluation by qPCR using validation cell lines}

We evaluated the expression profiling of the 19 genes using four validation cell lines (RERF-LC-MS [AD], LC$1 /$ sq [SQ], 86-2 [LC], and MS-1-L [SC]) and the normal control (MRC-9). The results of expression profiling are shown in Figure 1. The validation AD cell line showed similar upregulation in the same 12 genes $(A M Y 2 A$, BEX1, CDH1, CSTA, DUSP4, FOXG1, IGSF3, INADL, ISL1, MALL, S100P, and SLCO4A1) and downregulation in $P L A U$. The validation SQ cell line showed similar upregulation in 10 genes $(A M Y 2 A, B E X 1, C D H 1$, HMGA1, IGSF3, INADL, ISL1, MALL, RAB25 and $S L C O 4 A 1)$ and downregulation in PLAU. The validation LC cell line showed similar upregulation in 10 genes (AMY2A, CDH1, FOSL1, HMGA1, IGSF3, ISL1, MALL, $R A B 25, S 100 A 2$, and SLCO4A1). The validation SC cell line showed similar upregulation in the same nine genes (AMY2A, BEX1, FOXG1, IGSF3, INADL, ISL1, RAB25, $S L C O 4 A 1$, and STMN1) and downregulation in TGM2. Thus, the concordance rates were $100 \%$ for the AD, and $\mathrm{SC}$ validation lines, 92\% (11/12) for the SQ line, $77 \%$ (10/13) for the LC line, and 92\% (44/48) overall. CSTA, DUSP4, and S100P were upregulated consistently in only AD cells, and FOSL1 and S1OOA2 were upregulated in only LC cells. STMN1 was upregulated and TGM2 was downregulated in only SC cells.

\section{Principal component analysis (PCA)}

To classify the four histopathological subtypes by PCA we tried and selected various set of qPCR results from 19 genes. The four histopathological subtypes were 

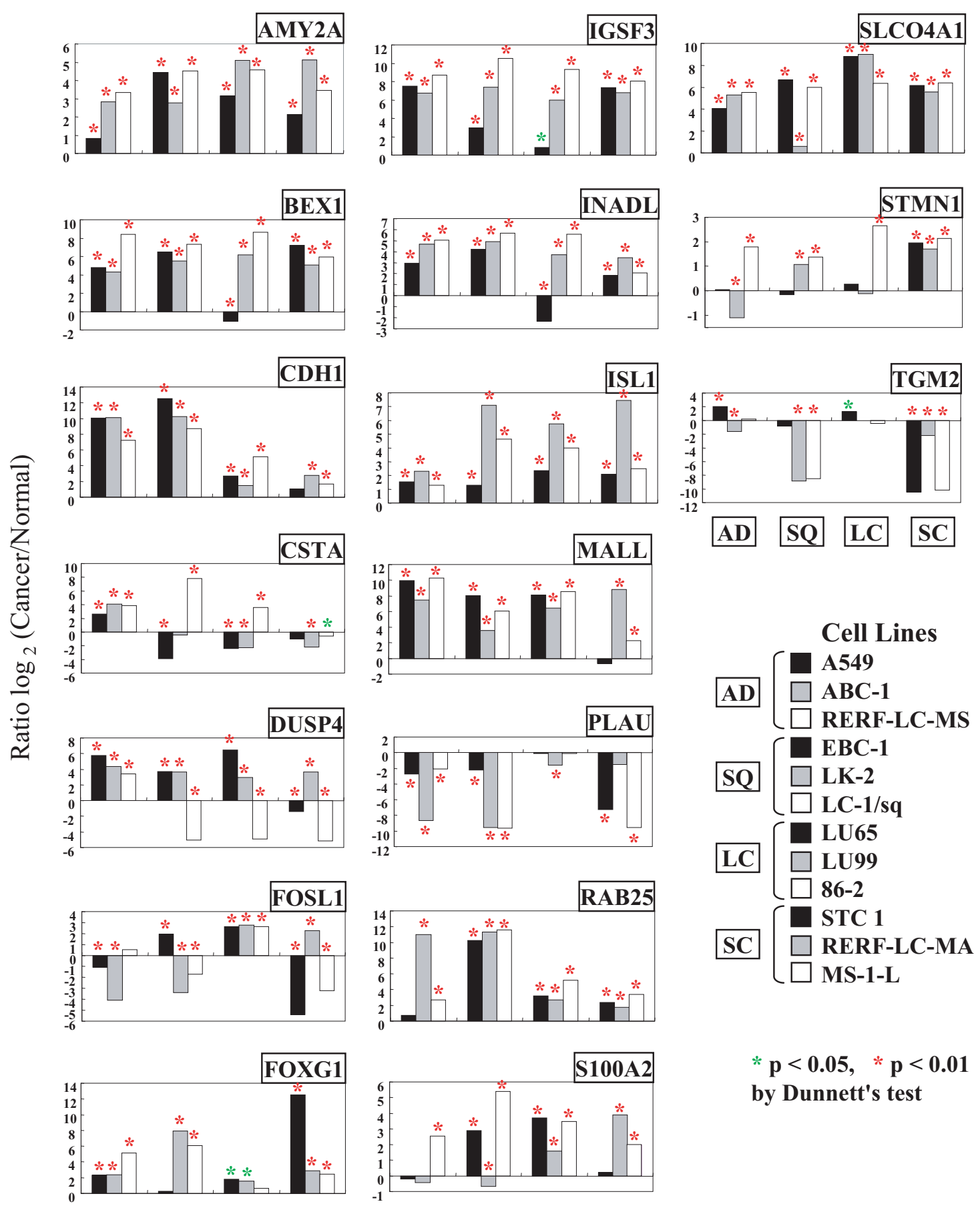

by Dunnett's test
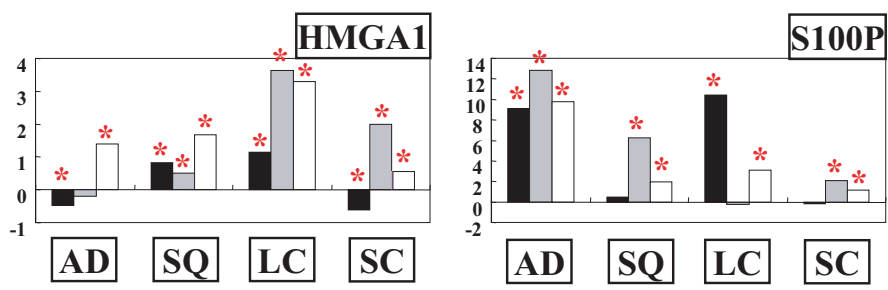

Figure 1 Quantification and validation of $\mathbf{1 9}$ genes by qPCR. The expression of 19 genes in 8 test lung cancer cell lines (black and gray) and four validation cell lines (white) was quantified by GPCR and compared to a normal control cell line (MRC-9). 
optimally classified by PCA using 12 genes $(A M Y 2 A$, CDH1, FOXG1, IGSF3, ISL1, MALL, PLAU, RAB25, S100P, SLCO4A1, STMN1, and TGM2) in the eight test cell lines, with the loading number of components 1,2 , and 3 (PC1, PC2, and PC3; 3 dimensions) shown in Figure 2(A). Using the same frame, the four subtypes were also classified using all 12 test and validation cell lines, as shown in Figure 2(B). Figure 2(B) shows that the four subtypes were divided into two prominent groups corresponding to positive $\mathrm{PC} 2$ values (AD and $\mathrm{SQ}$ ) and negative $\mathrm{PC} 2$ values ( $\mathrm{LC}$ and $\mathrm{SC}$ ). In Figure 2(C), which shows the principal component for the cell lines (PC1, $\mathrm{PC} 2$, and $\mathrm{PC} 3$ ), two of the three AD cells lines were close, whereas the third cell line was close in PC2 to the other two cell lines, but separate in PC1 and PC3. Specifically, A549 and RERF-LC-MS cells were close in PC1, $\mathrm{PC} 2$, and $\mathrm{PC} 3$, whereas $\mathrm{ABC}-1$ cells showed a distinct, small negative value in PC3. Two SQ cell lines, EBC-1 and LK-2, were close in PC1, PC2, and PC3, whereas one SQ cell line, LC-1/sq, showed a distinct, large positive value in PC1 and PC3. Two close AD cell lines and two close SQ cell lines were different in PC3 with a positive value in $A D$ and a negative value in $S Q$. $A B C-1$ (AD) and LC-1/sq (SQ) were also separated in different directions. Two LC cell lines, LU65 and LU99, were close in PC1, PC2, and PC3, whereas one LC cell line, 86-2, showed intermediate separation in PC1 and PC3. The three SC cell lines were close to each other. Two close LC cell lines and three SC cell lines were different in $\mathrm{PC} 1$, with a negative value for $\mathrm{LC}$ and a positive value for SC. The 86-2 cell line (LC) was different in PC3 from the SC cell lines. Subtypes were characterized by PC1-3 and loading number. In Figure 2(D), which shows the loading number for genes, $\mathrm{PC} 1$ was negatively correlated with the expression of PLAU, SLCO4A1, and TGM2, and positively correlated with IGSF3, STMN1, FOXG1, and RAB25. PC2 was negatively correlated with the expression of STMN1, AMY2A, and ISL1, and positively correlated with $S 100 P, R A B 25$, and $C D H 1$. PC3 was negatively correlated with the expression of $R A B 25$ and $A M Y 2 A$, and positively correlated with TGM2, $M A L L$, and IGSF3.

\section{Gene networks and gene pathways}

To further understand the biological networks of the 19 genes, we next analyzed their biological interactions using the Ingenuity Pathways Analysis (IPA) tool. Ten networks were extracted from each cancer cell line. Table 2 shows a major network (network 1) that contained 12 of the 19 candidate genes (AMY2A, BEX1, CDH1, DUSP4, FOSL1, FOXG1, HMGA1, ISL1, PLAU, S100P, STMN1, and TGM2), including 7 of the 12 PCA genes (CDH1, FOXG1, ISL1, PLAU, S10OP, STMN1, and TGM2), using ABC-1 cells as a representative cell line. The other 11 cell lines showed a similar network 1 , with various gene-specific increases and decreases and with slightly different top functions. The other nine networks were smaller (not shown). Figure 3 shows the gene networks of 11 of the 12 PCA genes (except SLCO4A1) based on IPA results. Link from SLCO4A1 to the present gene networks was not extracted by IPA. The connection including CDH1, PLAU, and SMAD4 suggested to be related to cell adhesion by IPA. The connection including TGM2, IL1B, and PLAU and the connection including RAB25, SNAI1, and CDH1 were suggested to be related to tumor invasion by IPA. STMN1 was suggested to influence cell motility, and S100P was suggested to be associated with cell growth by IPA.

\section{Immunohistochemistry}

Routine immunohistochemical studies were performed in four test cell lines (A549, EBC-1, LU65, and STC 1) and the control (MRC-9), to define their histopathological classification (Figure 4). S100P protein was expressed in the cytoplasm of A549 and LU65 cells. RAB25 protein was expressed in the cytoplasm of EBC-1 cells. These results were consistent with the gene expression data for S100P and RAB25 (Figure 1).

\section{Discussion}

We compared four histopathological subtypes of 12 lung cancer cell lines using a statistical processing method, PCA, which is based on gene expression profiling determined by qPCR. Four subtypes were optimally classified by PCA using 12 genes (AMY2A, CDH1, FOXG1, IGSF3, ISL1, MALL, PLAU, RAB25, S100P, SLCO4A1, $S T M N 1$, and TGM2) from the 19 candidate genes shown in Figure 1. PCA analysis revealed that the loading number of component 1 (PC1) was negatively correlated with the expression of PLAU, SLCO4A1, and TGM2, and positively correlated with IGSF3, STMN1, FOXG1, and RAB25. The loading number of component 2 (PC2) was negatively correlated with the expression of $S T M N 1, A M Y 2 A$, and $I S L 1$, and positively correlated with $S 100 P, R A B 25$, and $C D H 1$. The loading number of component 3 ( $\mathrm{PC} 3$ ) was negatively correlated with the expression of RAB25 and $A M Y 2 A$, and positively correlated with TGM2, MALL, and IGSF3. The four subtypes were divided into two prominent groups with $\mathrm{PC} 2$, corresponding to positive $\mathrm{PC} 2$ values $(\mathrm{AD}$ and $\mathrm{SQ}$ ) and negative $\mathrm{PC} 2$ values (LC and SC). Because $\mathrm{PC} 2$ was positively correlated with the expression of $C D H 1$, $S 100 P$, and $R A B 25$, these genes may be significant in the classification of the four subtypes. Three SC cell lines were close to each other in PC1, PC2, and PC3. As the presence of subclasses in AD and SQ clinical tissues was suggested $[6,7,18]$, it was probable that there was some diversity in the present AD and SQ cell lines. Gene expression of these 12 genes was generally consistent with some exceptions in the four subtypes. Even when 


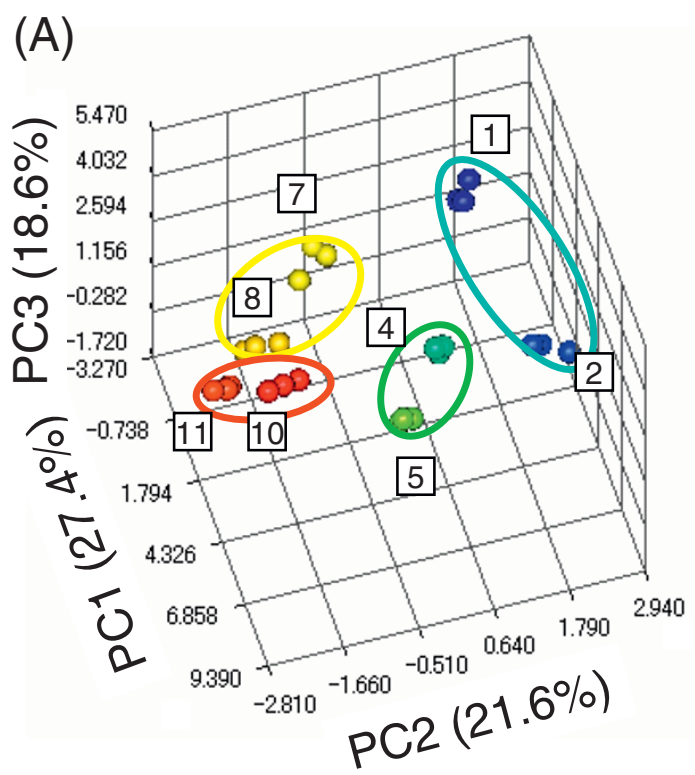

(C) Principal component for cell lines

\begin{tabular}{|r|l|r|r|r|}
\hline No & Strains & \multicolumn{1}{l|}{ PC1 } & PC2 & \multicolumn{1}{l|}{ PC3 } \\
\hline 1 & A549(AD) & -0.63 & 1.56 & 3.23 \\
\cline { 2 - 5 } 2 & ABC-1(AD) & 0.94 & 2.63 & -0.98 \\
\cline { 2 - 5 } 3 & RERF-LC-MS(AD) & 1.57 & 0.05 & 3.92 \\
\hline 4 & EBC-1(SQ) & -0.72 & 1.20 & -1.59 \\
\cline { 2 - 5 } 5 & 2.34 & 0.26 & -0.98 \\
\cline { 2 - 5 } 6 & LK-2(SQ) & 9.05 & 0.51 & 5.19 \\
\hline 7 & LC-1/sq(SQ) & -3.00 & -0.41 & 0.30 \\
\cline { 2 - 5 } 8 & LU65(LC) & -1.78 & -1.39 & -0.99 \\
\cline { 2 - 5 } 9 & LU9-2(LC) & 2.83 & -2.59 & 3.53 \\
\hline 10 & STC 1(SC) & 2.51 & -1.62 & 1.24 \\
\cline { 2 - 5 } 11 & RERF-LC-MA(SC) & 0.35 & -2.24 & -0.22 \\
\cline { 2 - 5 } 12 & MS-1-L(SC) & 2.22 & -1.28 & 1.01 \\
\hline
\end{tabular}

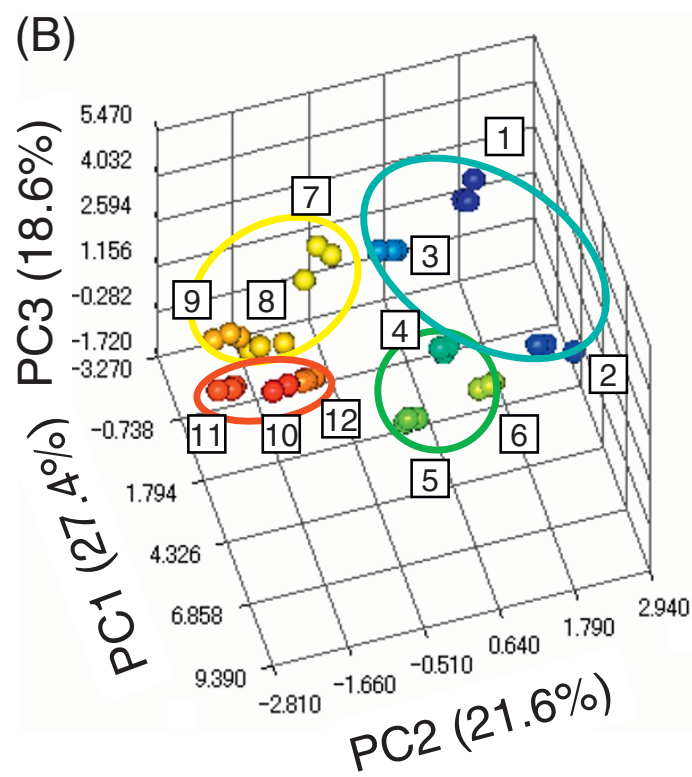

(D) Loading number for genes

\begin{tabular}{|l|c|}
\hline & PC1 \\
\hline PLAU & -0.83 \\
\hline SLCO4A1 & -0.76 \\
\hline TGM2 & -0.58 \\
\hline MALL & -0.33 \\
\hline AMY2A & -0.32 \\
\hline CDH1 & -0.04 \\
\hline S100P & 0.08 \\
\hline ISL1 & 0.29 \\
\hline RAB25 & 0.48 \\
\hline FOXG1B & 0.55 \\
\hline STMN1 & 0.57 \\
\hline IGSF3 & 0.72 \\
\hline
\end{tabular}

\begin{tabular}{|l|c|}
\hline & PC3 \\
\hline RAB25 & -0.54 \\
\hline AMY2A & -0.51 \\
\hline CDH1 & -0.37 \\
\hline ISL1 & -0.28 \\
\hline SLCO4A1 & -0.20 \\
\hline S100P & -0.18 \\
\hline PLAU & 0.00 \\
\hline STMN1 & 0.21 \\
\hline FOXG1B & 0.31 \\
\hline IGSF3 & 0.52 \\
\hline MALL & 0.69 \\
\hline TGM2 & 0.72 \\
\hline
\end{tabular}

Figure 2 Principal component analysis of cell lines with 12 genes based on qPCR data. PCA differentiates four histopathological subtypes by three-dimensional expression clustering. The values of triplicate QPCR assays for each sample were analyzed. Results of PCA are shown in the three-dimensional contribution scores for component numbers 1, 2, and 3 (PC1, PC2, and PC3), which discriminate the four histopathological clusters. Data are shown for the eight test cell lines alone (A) and in combination with the four validation cell lines (B). The contribution scores were produced by conversion from each eigenvector value.

Table 2 IPA network 1 of ABC-1 cell

\begin{tabular}{|c|c|c|c|c|}
\hline Cell & Genes in network & Score & Focus genes & Top functions \\
\hline$A B C-1$ & $\begin{array}{l}\text { AGER, Ap1, } \uparrow \text { BEX1, } \\
\text { CDC42EP5, } \uparrow \text { CDH1, Ck2, } \\
\text { deoxycholate, } \uparrow \text { DUSP4, ERK, } \\
\downarrow \text { FOSL1, } \uparrow \text { FOXG1, FSH, } \\
\text { FXYD5, GDF15, HMGA1, } 18 \text { r, } \\
\uparrow \text { ISL1, Jnk, LCN2, MAD2L2, } \\
\text { Mapk, MGAT3, MKP2/5, NFkB, } \\
\text { PDGF BB, PI3K, } \downarrow \text { PLAU, } \\
\text { PTPRF, PVR, RAGE, S100A1, } \\
\text { S100P, SLC12A6, } \downarrow \text { STMN1, } \downarrow \\
\text { TGM2 }\end{array}$ & 28 & 11 & $\begin{array}{l}\text { Cancer, Cellular Movement, } \\
\text { Cellular Growth and } \\
\text { Proliferation }\end{array}$ \\
\hline
\end{tabular}

Biologically relevant network 1 extracted by IPA is shown for ABC-1 cell as a representative. $\uparrow$ marks represent upregulated genes and $\downarrow$ marks downregulated genes. genes. 


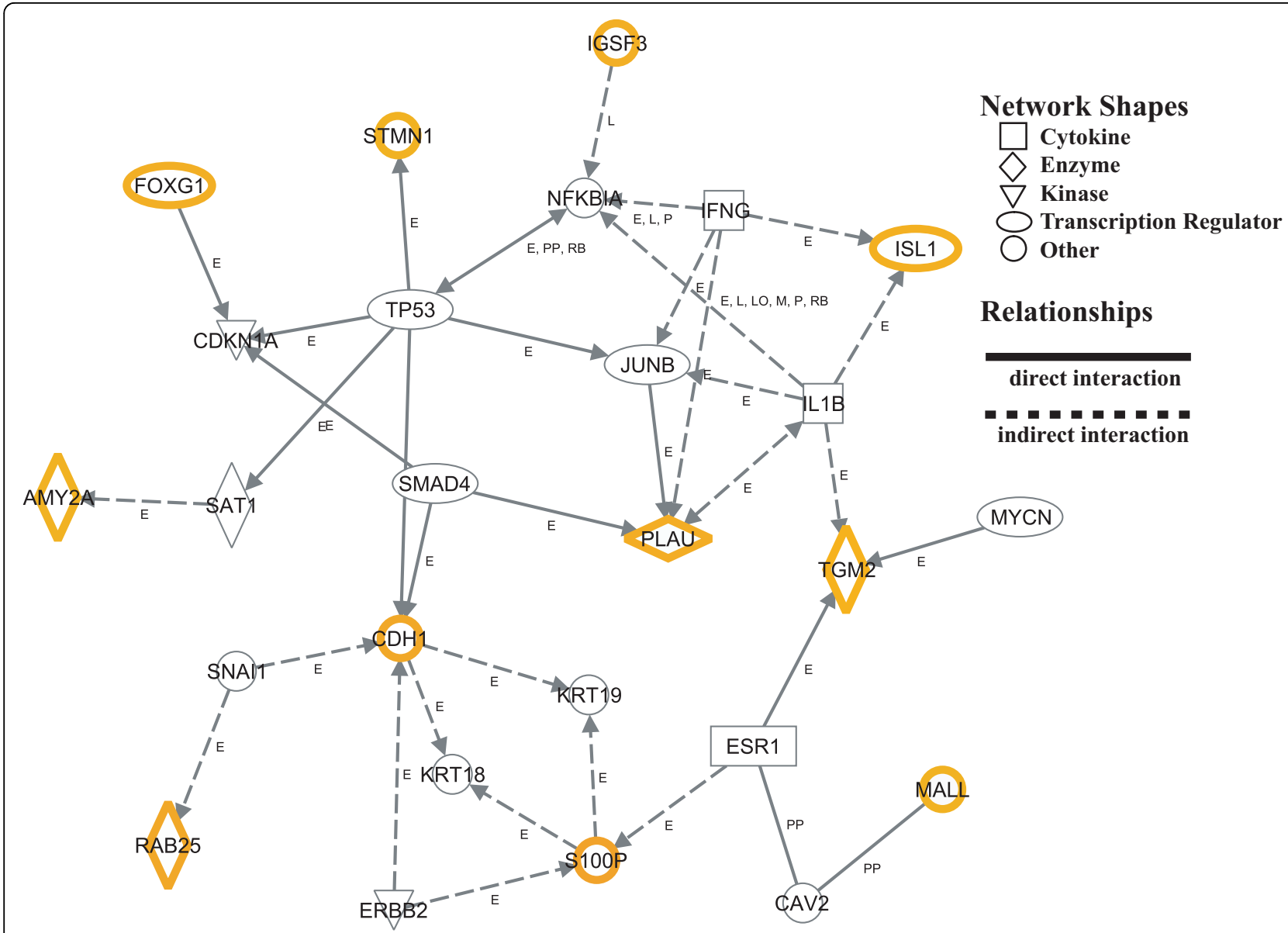

Figure 3 Gene networks and pathways of $\mathbf{1 1}$ genes from PCA analysis. The network was analyzed using Ingenuity Pathways Analysis software and is displayed graphically as nodes (genes/gene products) and edges (the biological relationships between the nodes). Nodes are displayed using shapes that represent the functional class of the gene product, as indicated in the key. Edges are displayed with labels that describe the nature of the relationship between the nodes ( $E$, expression; $L$, proteolysis; LO, localization; $M$, biochemical modification; $P$, phosphorylation/dephosphorylation; PP, protein-protein binding; RB, regulation of binding).

gene expression was not fully consistent among the subtypes, PCA with the present 12 genes could be used effectively to classify the four subtypes.

Using DNA microarrays and qPCR, Kuner et al. [19] recently compared gene expression in $42 \mathrm{AD}$ and $18 \mathrm{SQ}$ clinical tumor samples and systematically analyzed their expression patterns using gene ontology. This group identified 14 tight junction genes and 9 epithelialmesenchymal transition genes that were upregulated or downregulated in AD samples, SQ samples, or both. Among these genes, the epithelial-mesenchymal transition gene $C D H 1$, which codes for E-cadherin, was upregulated in both $\mathrm{AD}$ and $\mathrm{SQ}$ samples. We also examined gene ontology. Although our overall results were unclear, our data suggest that $C D H 1$ is associated with cell adhesion, and that the AD and SQ cell lines are associated with greater cell adhesion, while LC and SC cell lines are associated with weaker cell adhesion. Taken together, these studies demonstrate a remarkable upregulation of $C D H 1$ in $\mathrm{AD}$ and SQ cells, but not LC and SC cells, making this a candidate marker for differentiating lung cancer subtypes. $C D H 1$ was the only gene studied in both the Kuner et al. report and in ours.

Using cDNA microarrays and gene ontology, Inamura et al. [18] analyzed 48 SQ clinical tissue samples and classified them into two subclasses. Subclass A genes were related to processes such as cell proliferation and cell cycle progression, while subclass B genes were related to processes such as the MAPKKK cascade and apoptosis. They focused on 30 possible marker genes that were completely different from the 23 genes identified in the Kuner et al. report and the 12 genes we studied.

Using bioinformatics, Kim et al. [20] extracted differentially expressed lung cancer candidate genes from published data examined by SAGE method. Next, they used qPCR to compare candidate gene expression in 18 $\mathrm{AD}$ and $18 \mathrm{SQ}$ samples from microdissected clinical 


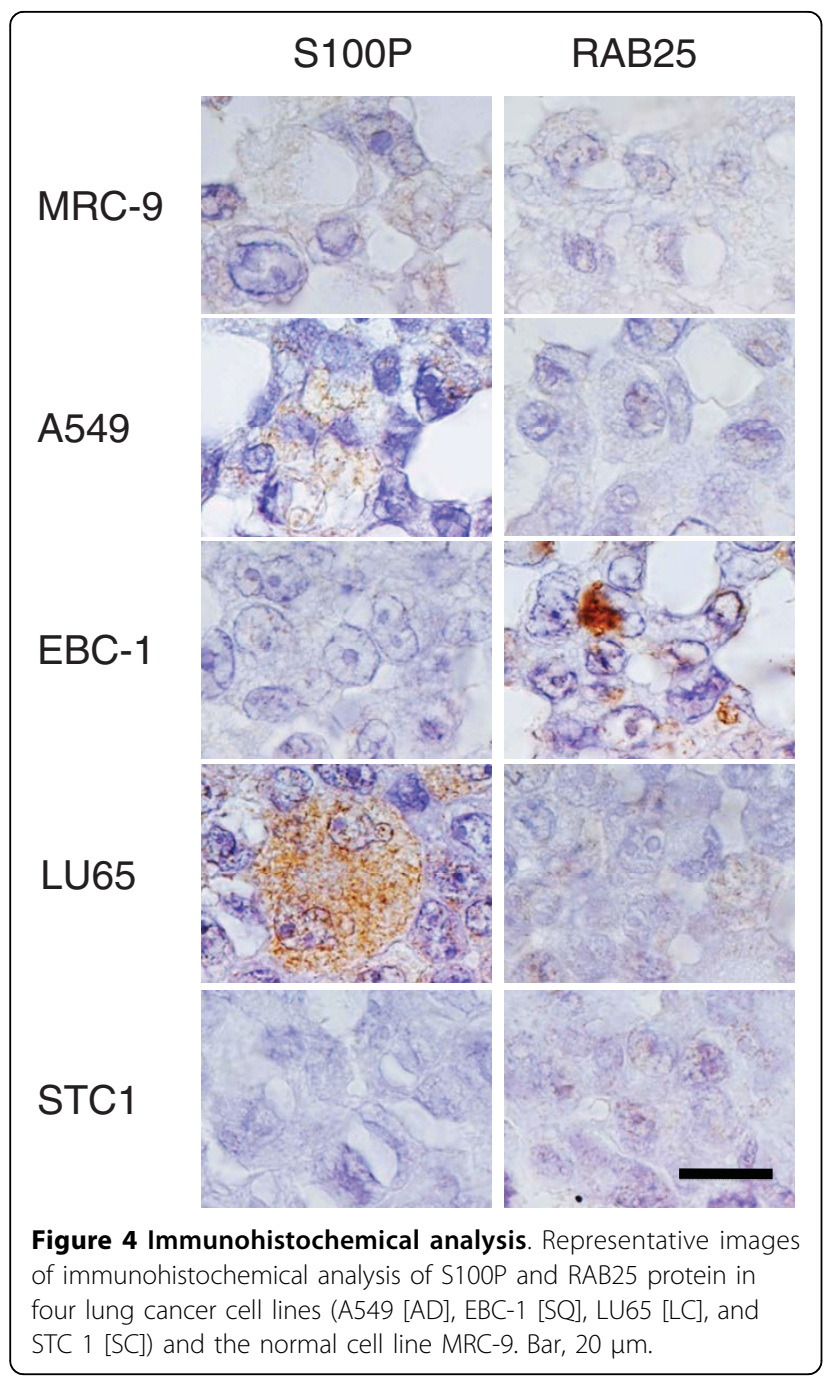

tissues. They extensively examined seven genes and identified two, $C B L C$ and $C Y P 24 A 1$, as novel candidate biomarkers for $\mathrm{AD}$ and SQ cells. They also suggested that $S 100 P$, which encodes $S 100$ calcium-binding protein $\mathrm{P}$, may be a good biomarker for AD cells. The expression ratio of $S 100 P$ in cancer/normal cells was high in AD samples and low in SQ samples. In our study, all three AD cell lines showed a robust increase in S100P expression, while the three SQ cell lines showed less or no increase. Taking our data and the Kim et al. data together, the remarkable and specific upregulation of S100P in AD cells suggests that this is a candidate marker for distinguishing the AD subtype. Although the Kim and Kuner groups both analyzed AD and SQ samples, their gene sets (7 and 30, respectively) were nonoverlapping.

Identification of molecular markers often leads to important clinical applications, such as earlier diagnosis, better prognosis, and more effective drug targeting.
Although numerous papers examining lung cancer tissues and/or lung cancer cell lines using DNA microarrays and/or qPCR have been published e.g. [1-3,6-10,19,20], lung cancers still lack reliable molecular markers [20]. The genes examined varied between paper, and the results were not necessarily consistent. This variability may result from technical limitations, differences in methodology, and the broad biological heterogeneity of lung cancers themselves. Continued accumulation of data will help resolve this question. The studies described were conducted primarily with AD and SQ samples. Many fewer studies looked at LC and SC samples, and direct comparison of all four histopathological subtypes using the same method(s) was rare. Our study is unique because we examined 12 lung cancer cell lines representing all four subtypes, and we used both qPCR and PCA of 12 genes (AMY2A, CDH1, FOXG1, IGSF3, ISL1, MALL, PLAU, RAB25, S100P, $S L C O 4 A 1, S T M N 1$, and TGM2). Although none of these 12 genes represent novel candidate markers because they were all selected from earlier microarray studies [1-3,6-10], this is the first report that systematically analyzed them together in all four subtypes.

The gene network was analyzed using Ingenuity Pathways Analysis software and is displayed graphically in Figure 3. The first connection including CDH1, PLAU, and SMAD4 was suggested to be related to cell adhesion $[21,22]$. It was reported that $S M A D 4$ reduced the expression level of endogenous PLAU [21] and induced $C D H 1$ expression [22]. The second connection including RAB25, SNAI1, and CDH1 was suggested to be related to tumor invasion. It was reported that $R A B 25$ enhanced the ability of tumor cells to invade the extracellular matrix [23]. The first and second connection may be applicable to AD and SQ cell lines in this study. It was reported that STMN1 influenced cell motility [24] and S100P was associated with cell growth [25]. STMN1 and S100P may work in SC cell lines and AD cell lines, respectively, in this study. The third connection including TGM2, IL1B, and PLAU was suggested to be tumor invasion. It was reported that $I L 1 B$ increased the expression level of TGM2 [26], which might be involved in establishing a barrier to tumor spreading [27]. The third connection may not be effective in cell lines in this study, because TGM2 was rather downregulated in this study.

Six of the genes analyzed (CDH1, PLAU, RAB25, S10OP, STMN1, and TGM2) have attracted recent attention relating to therapeutic drug sensitivity and prognosis. In gene expression profiling studies of lung cancer cell lines to study therapeutic drug sensitivity, $P L A U$ and $C D H 1$ have been suggested as novel biomarkers of cetuximab sensitivity [28], and TGM2 was suggested as a potential marker of doxorubicin sensitivity 
[27]. STIMN1 was reported to be a novel therapeutic target for anticancer activity [29]. Additionally, RAB25 may be linked to tumor aggressiveness and metastasis [23], and $S 100 P$ may be a diagnostic marker of nonsmall-cell lung cancer [30,31]. PLAU has also been examined in relation to lung cancer prognosis [32]. The set of 12 well-characterized cell lines described in this study, representing the four histopathological subtypes, should prove useful for screening therapeutic drugs and their effects on specific genes.

We performed additional immunohistochemical studies to examine S100P and RAB25 (Figure 4). The results were generally consistent with the gene expression data (Figure 1). In immunostained tumor tissues, AD cells showed immunostaining of S100P in the cytoplasm and the nucleus, while SQ cells showed immunostaining of RAB25 in the cytoplasm. The localization of S100P and RAB25 in tumor tissues was similar to that in cultured cells (data not shown).

Although DNA microarray technology is a powerful tool for characterizing gene expression on a genome scale, issues of reliability, reproducibility, and the correlation of data across different DNA microarrays still need to be addressed. Recently, qPCR was described as being simpler and more reliable than DNA microarrays [13]. Our experiments confirmed that qPCR was simpler, more reproducible, and more reliable than DNA microarrays. In the future, identification of reliable marker genes will hopefully allow for the development of automatic $\mathrm{qPCR}$ systems for routine clinical cancer diagnosis.

We examined the characteristics of four histopathological subtypes in lung cancer cell lines using both statistical analysis and biological network analysis. In the future, studies with cultured lung cancer cells should improve our ability to predict the response of different lung cancer types to specific therapeutic regimens.

\section{Conclusions}

Our results showed that the four histopathological subtypes, represented by 12 lung cancer cell lines, were well characterized by qPCR and PCA using 12 genes: AMY2A, CDH1, FOXG1, IGSF3, ISL1, MALL, PLAU, RAB25, S100P, SLCO4A1, STMN1, and TGM2. Based on their upregulation and the results of the PCA analysis, $S 100 P$ and $C D H 1$ were identified as candidate markers for $\mathrm{AD}$ tumors and for $\mathrm{AD}$ and $\mathrm{SQ}$ tumors, respectively.

\section{Methods}

\section{Cell lines and RNA isolation}

The human lung cancer cell lines ABC-1 (AD), RERFLC-MS (AD), EBC-1 (SQ), LK-2 (SQ), LC-1/sq (SQ), LU65 (LC), LU99 (LC), STC 1 (SC), RERF-LC-MA (SC),
MS-1-L (SC), and MRC-9 (normal control lung cell line) were purchased from the Japanese Collection Research Resources Bank (JCRB, Osaka Japan). The 862 (LC) lung cancer cell line was purchased from Riken Bioresource Center (Tsukuba, Japan), and the A549 (AD) lung cancer cell line was a generous gift provided by Dr. Akira Yasui of Tohoku University (Sendai, Japan). Total RNA samples were isolated from each cultured cell line using Micro Smash MS-100 (Tomy Digital Biology Co., Ltd. Tokyo) and QuickGene-800 (Fujifilm, Tokyo). RNA quality assurance was performed by measuring the $260: 280 \mathrm{~nm}$ ratio with a spectrophotometer (NanoDrop Technologies, LLC, Wilmington, DE, USA) and by gel electrophoresis using the Bioanalyzer and Agilent RNA 6000 Nano kit (Agilent Technologies Inc., Santa Clara, CA, USA).

\section{DNA microarray design and production}

The 100 candidate marker genes, which were selected based on previous reports [1-3,6-10], are shown in additional file 1http://www.chem.aoyama.ac.jp/Chem/ ChemHP/Furihatalab/. Synthesis of newly designed probes (Japan Patent No. 2007-234363) was outsourced to Invitrogen Corp. (Carlsbad, CA, USA). The probes were spotted onto a GeneSlide platform (Toyo Kohan Co., Ltd. Tokyo) using a Genex Arrayer Type-M (Kaken Geneqs, Inc., Chiba, Japan). GeneSlides were prehybridized at $80^{\circ} \mathrm{C}$ for 1 hour, washed in $2 \times \mathrm{SSC} / 0.2 \% \mathrm{SDS}$ and then ultrapure water, and then dried by centrifugation.

\section{cDNA synthesis and gene expression profiling by DNA} microarray

Alexa-labeled target cDNA was prepared from $20 \mu \mathrm{g}$ total RNA using a SuperScript Plus Indirect cDNA System kit (Invitrogen Corp., Carlsbad, CA, USA). cDNA obtained from cancer cell lines was labeled with Alexa 555 , and cDNA obtained from the control cell line was labeled with Alexa 647. The two Alexa-labeled cDNA samples were mixed and hybridized to a single DNA microarray that was then scanned in a DNA microarray scanner (FLA-8000, Fujifilm). To identify upregulated and downregulated genes, the ratio of relative intensities of the two fluorophores (Alexa 555: Alexa 647) was calculated after global normalization using ArrayGauge (Fujifilm). DNA microarray array data were deposited into the Center for Information Biology Gene Expression Database (CIBEX; accession: CBX 100).

\section{Quantification of genes using qPCR}

cDNA was prepared from $2.5 \mu \mathrm{g}$ total RNA using the SuperScript first-strand synthesis system from an RTPCR kit (Invitrogen Corp., Carlsbad, CA, USA). qPCR amplifications were performed with triplicate assays using the SYBR Green I assay in an Opticon 2 thermal cycler (MJ Research, Inc., Waltham, MA, USA). The reactions were carried out in a 96-well plate in $20-\mu \mathrm{l}$ reactions containing $2 \times$ SYBR Green Master Mix 
(Applied Biosystems, Lincoln Centre Drive Foster City, CA, USA), 2 pmol of each forward and reverse primer, and a cDNA template corresponding to $400 \mathrm{pg}$ total RNA. The primer sequences and $\mathrm{Ct}$ values of the 19 candidate genes and GAPDH (a housekeeping gene as an internal control) are shown in Table 3. SYBR Green PCR conditions were $95^{\circ} \mathrm{C}$ for 10 minutes, followed by 45 cycles of $95^{\circ} \mathrm{C}$ for 10 seconds, $58^{\circ} \mathrm{C}$ for 50 seconds, and $72^{\circ} \mathrm{C}$ for 20 seconds. In each assay, a standard curve was calculated concurrently with the examined samples. In the preliminary experiment, the group expressing the highest amount of product was selected for each gene and used as the standard sample in the subsequent assay. Each standard curve consisted of six serial dilutions $(1,1 / 5,1 / 25,1 / 125,1 / 625$, and $1 / 3125)$ of the selected standard cDNA for each gene. The relative quantitative value of each sample was determined with the 1/25-diluted cDNA and was normalized to GAPDH as described previously [33]. Relative GAPDH expression in the experimental cell lines is shown in Figure 5.

\section{Gene pathways, networks, and ontology analysis}

Biological networks were generated with Ingenuity Pathways Analysis 7.0 (IPA), a web-based application http:// www.Ingenuity.com that enables the visualization and analysis of biologically relevant networks to enable the discovery, visualization, and exploration of therapeutically relevant networks as described previously [33]. Ontology analysis was performed with IPA and http:// geneontology.org/.

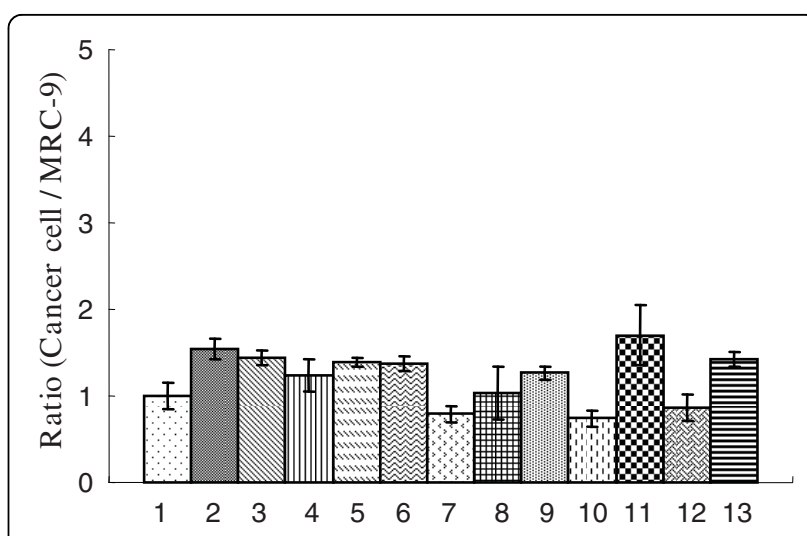

Figure 5 Relative expression of GAPDH. Total RNA was extracted from each of the 12 lung cancer cell lines and reverse-transcribed to produce CDNA. GAPDH expression was determined by qPCR in triplicate assays. Results are shown as the mean \pm S.D. Numbers indicate cell lines, 1: MRC-9 [normal control], 2: A549 [AD], 3: ABC-1 [AD], 4: RERF-LC-MS [AD], 5: EBC-1 [SQ], 6: LK-2 [SQ], 7: LC-1-Sq [SQ], 8: LU65 [LC], 9: LU99 [LC], 10: 86-2 [LC], 11: STC 1 [SC], 12: RERF-LCMA [SC] and 13: MS-1-L [SC].

\section{Immunohistochemistry}

Routine immunohistochemistry was performed using formalin-fixed, paraffin-embedded sections as described in the manufacturer's protocol. We could obtain only RAB25 and S100P antibodies. The following antibodies, dilutions, and pretreatment conditions were used: antiRAB25 (1:100), trypsin pretreatment; Abnova Corporation, Taipei, Taiwan) and anti-S100P (polyclonal rabbit

Table 3 Primer sequences of 20 genes examined in the study

\begin{tabular}{|c|c|c|c|c|}
\hline No. & Symbol & $\mathrm{Ct}$ & Left & Right \\
\hline 1 & $A M Y 2 A$ & $25-31$ & GGGTTCGTATTTATGTGGATGC & GGGTTCGTATTTATGTGGATGC \\
\hline 2 & BEX1 & $21-31$ & GCCTAGAGGAAATCGTAGGCGGTTC & CTCTCATCCTTGCCTGTGGTTCTCC \\
\hline 3 & $\mathrm{CDH} 1$ & $22-33$ & TGGGCAGCTATCCAGTGACTTGTTC & CTGTCTITGGCTGCAGCACTITAGG \\
\hline 4 & CSTA & $25-35$ & CACTTTGGTTCCAGCATCCTGTC & ACAATCTCCTGGATTTCTGGAGTG \\
\hline 5 & DUSP4 & $20-29$ & CTGCTTCTCAGTGGCAACAAAC & CCGTAGCATGCAGATGTCAAGG \\
\hline 6 & FOSL1 & $18-22$ & TAAGGCGCGAGCGGAACAAG & TCGCTGCAGCCCAGATTTCTC \\
\hline 7 & FOXG1 & $23-36$ & GCGCCAGCAGCACTITGAGTTAC & TGGTTGTTGCCCAGCGAGTTC \\
\hline 8 & GAPDH(GAPD) & $21-22$ & AGTAGAGGCAGGGATGATGTTC & CTITGGTATCGTGGAAGGACTC \\
\hline 9 & HMGA1 & $17-20$ & GCGAAGTGCCAACACCTAAGAGACC & CCTTGGTTTCCTTCCTGGAGTTGTG \\
\hline 10 & IGSF3 & $24-35$ & GACCTCATTGCGCATTGTCTAC & CATGTCCTAGAATGCGCCTAG \\
\hline 11 & INADL & $23-31$ & AGAATGGACTTGGACTCAGCCTTGC & CATCTCCAATACGCATTCGTCCATC \\
\hline 12 & ISL1 & $24-33$ & GTACGGGATCAAATGCGCCAAG & AGGCCACACAGCGGAAACACTC \\
\hline 13 & MALL(BENE) & $21-33$ & CATTACATCCGTGGATTCTCC & GTGCTTAAGAGAATGTGAGGG \\
\hline 14 & PLAU & $21-31$ & CGCTGCTCCCACATTGGCTAAG & TGTGCATGGGTGAAGGGAGAGC \\
\hline 15 & RAB25 & $21-33$ & TGATCGGCGAATCAGGTGTGG & CAACATCACAGTGCGGGTGGAG \\
\hline 16 & S100A2 & $22-27$ & GCAGCCTGGATGAGAACAGTGACC & CAGCCCTGGAAGAAGTCATTGCAC \\
\hline 17 & S100P & $18-32$ & GTCTGAATCTAGCACCATGACG & GGAAGCCTGGTAGCTCCTTC \\
\hline 18 & SLCO4A1 & $22-32$ & TCCATCTGGCTCCTGCTGAAGAAC & GCTTCTGAGGCACTCAGGCTGAAC \\
\hline 19 & STMN1 & $21-25$ & AAAGAACTGGAGAAGCGTGCCTCAG & CTGAATTTCCTCCAGGGAAAGATCC \\
\hline 20 & TGM2 & $21-36$ & CTGGTCACTAACCAACAAGGTTG & GAGCAGGAGATAAAGTCAAAGCTG \\
\hline
\end{tabular}

Ct: cycle threshold. 
anti-S100, (1:400), trypsin pretreatment; DakoCytomation, Copenhagen, Denmark).

\section{Statistical analysis}

For statistical analysis program, we performed a logarithmic $\left(\log _{2}\right)$ transformation of the data to stabilize the variance and the gene expression profile of each cancer cell line, which was normalized to the median gene expression level for the entire sample set. DNA microarray results of the eight test cell lines were analyzed with a Dunnett's test. qPCR results of the 19 candidate genes in 12 cell lines were analyzed with a Dunnett's test and principal component analysis (PCA). PCA was performed using both the PCA program in R (2.8.0; http:// www.r-project.org/) and Microsoft Office Excel 2003 (Microsoft, Redmond, WA, USA).

Additional file 1: One hundred genes examined in the present

study. Symbols, gene names and accession numbers are shown.

Click here for file

[ http://www.biomedcentral.com/content/supplementary/1475-2867-10-2S1.pdf]

\section{Acknowledgements}

This work was supported in part by Regional New Consortium R\&D Projects, The Ministry of Economy, Trade and Industry, Japan (C. Furihata), and a Grant-in-Aid from the Private School High-tech Research Center Program of the Ministry of Education, Culture, Sports, Science, and Technology, Japan (C. Furihata). We thank Dr. Takayuki Negishi, School of Science and Engineering, Aoyama Gakuin University, for collaboration with the Bioanalyzer experiment, and Dr. Kazuhiko Matsumoto, Torii Pharmaceutical Co. Ltd. for his advice on Dunnett's test.

\section{Author details}

'Department of Chemistry and Biological Science, School of Science and Engineering, Aoyama Gakuin University, Kanagawa 229-8558, Japan. ${ }^{2}$ Department of Chest Surgery, Niigata Rosai Hospital, Japan Labor Health and Welfare Organization, Niigata 942-8502, Japan. ${ }^{3}$ Department of Pathology, Niigata Rosai Hospital, Japan Labor Health and Welfare Organization, Niigata 942-8502, Japan.

\section{Authors' contributions}

TW: Experimental design, all experiments, interpretation of data, and manuscript preparation. TM: Experiments (cell culture and DNA microarray analysis). YD: Experiments (cell culture and quantitative real-time PCR analysis). YF: Experiments (cell culture and quantitative real-time PCR analysis). MI: Experiments (analysis with antibody). MK: Experiments (analysis with antibody). CF: Experimental design, conception, and manuscript preparation. All authors have read and approved the final manuscript.

\section{Competing interests}

The authors declare that they have no competing interests.

Received: 5 October 2009

Accepted: 21 January 2010 Published: 21 January 2010

\section{References}

1. Giordano TJ, Shedden KA, Schwartz DR, Kuick R, Taylor JM, Lee N, Misek DE, Greenson JK, Kardia SL, Beer DG, Rennert G, Cho KR, Gruber SB, Fearon ER, Hanash S: Organ-specific molecular classification of primary lung, colon, and ovarian adenocarcinomas using gene expression profiles. Am J Pathol 2001, 159:1231-1238.

2. Beer DG, Kardia SL, Huang CC, Giordano TJ, Levin AM, Misek DE, Lin L, Chen G, Gharib TG, Thomas DG, Lizyness ML, Kuick R, Hayasaka S, Taylor JM, lannettoni MD, Orringer MB, Hanash S: Gene-expression profiles predict survival of patients with lung adenocarcinoma. Nat Med 2002, 8:816-824.

3. Endoh H, Tomida S, Yatabe Y, Konishi H, Osada H, Tajima K, Kuwano H, Takahashi T, Mitsudomi T: Prognostic model of pulmonary adenocarcinoma by expression profiling of eight genes as determined by quantitative real-time reverse transcriptase polymerase chain reaction. J Clin Oncol 2004, 22:811-819.

4. Fan X, Shi L, Fang H, Harris S, Perkins R, Tong W: Investigation of reproducibility of differentially expressed genes in DNA microarrays through statistical simulation. BMC Proc 2009, 3(Suppl 2):S4.

5. Yauk $\mathrm{CL}$, Berndt $\mathrm{ML}$ : Review of the literature examining the correlation among DNA microarray technologies. Environ Mol Mutagen 2007, 48:38094.

6. Garber ME, Troyanskaya OG, Schluens K, Petersen S, Thaesler Z, PacynaGengelbach M, Rijn van de M, Rosen GD, Perou CM, Whyte RI, Altman RB, Brown PO, Botstein D, Petersen I: Diversity of gene expression in adenocarcinoma of the lung. Proc Natl Acad Sci USA 2001, 98:13784-13789.

7. Bhattacharjee A, Richards WG, Staunton J, Li C, Monti S, Vasa P, Ladd C, Beheshti J, Bueno R, Gillette M, Loda M, Weber G, Mark EJ, Lander ES, Wong W, Johnson BE, Golub TR, Sugarbaker DJ, Meyerson M: Classification of human lung cancer by mRNA expression profiling reveals distinct adenocarcinoma subclasses. Proc Natl Acad Sci USA 2001, 98:13790-13795.

8. Nacht M, Dracheva T, Gao Y, Fujii T, Chen Y, Player A, Akmaev V, Cook B, Dufault M, Zhang M, Zhang W, Guo M, Curran J, Han S, Sidransky D, Buetow K, Madden SL, Jen J: Molecular characteristics of non-small cell lung cancer. Proc Natl Acad Sci USA 2001, 98:15203-15208.

9. McDoniels-Silvers AL, Nimri CF, Stoner GD, Lubet RA, You M: Differential gene expression in human lung adenocarcinomas and squamous cell carcinomas. Clin Cancer Res 2002, 8:1127-1138.

10. Virtanen C, Ishikawa Y, Honjoh D, Kimura M, Shimane M, Miyoshi T, Nomura $\mathrm{H}$, Jones $\mathrm{MH}$ : Integrated classification of lung tumors and cell lines by expression profiling. Proc Natl Acad Sci USA 2002, 99:12357-12362.

11. Qin LX, Beyer RP, Hudson FN, Linford NJ, Morris DE, Kerr KF: Evaluation of methods for oligonucleotide array data via quantitative real-time PCR. BMC Bioinformatics 2006, 7:23.

12. Nardon E, Donada M, Bonin S, Dotti I, Stanta G: Higher random oligo concentration improves reverse transcription yield of CDNA from bioptic tissues and quantitative RT-PCR reliability. Exp Mol Pathol 2009, 87:146-51.

13. Provenzano M, Mocellin S: Complementary techniques: validation of gene expression data by quantitative real time PCR. Adv Exp Med Biol 2007, 593:66-73.

14. Ju Z, Kapoor M, Newton K, Cheon K, Ramaswamy A, Lotan R, Strong LC, Koo JS: Global detection of molecular changes reveals concurrent alteration of several biological pathways in nonsmall cell lung cancer cells. Mol Genet Genomics 2005, 274:141-54.

15. Waagmeester AS, Kelder T, Evelo CT: The role of bioinformatics in pathway. Genes Nutr 2008, 3:139-142.

16. Braakhuis $\mathrm{BJ}$, Leemans $\mathrm{CR}$, Brakenhoff RH: Using tissue adjacent to carcinoma as a normal control: an obvious but questionable practice. $J$ Pathol 2004, 203:620-621.

17. Braakhuis BJ, Tabor MP, Kummer JA, Leemans CR, Brakenhoff RH: A genetic explanation of Slaughter's concept of field cancerization: evidence and clinical implications. Cancer Res 2003, 63:1727-30.

18. Inamura $K$, Fujiwara $T$, Hoshida $Y$, Isagawa $T$, Jones MH, Virtanen $C$, Shimane M, Satoh Y, Okumura S, Nakagawa K, Tsuchiya E, Ishikawa S, Aburatani $H$, Nomura $H$, Ishikawa $Y$ : Two subclasses of lung squamous cell carcinoma with different gene expression profiles and prognosis identified by hierarchical clustering and non-negative matrix factorization. Oncogene 2005, 24:7105-13.

19. Kuner R, Muley T, Meister M, Ruschhaupt M, Buness A, Xu EC, Schnabel P, Warth A, Poustka A, Sültmann H, Hoffmann H: Global gene expression analysis reveals specific patterns of cell junctions in non-small cell lung cancer subtypes. Lung Cancer 2009, 63:32-8.

20. Kim B, Lee HJ, Choi HY, Shin Y, Nam S, Seo G, Son DS, Jo J, Kim J, Lee J, Kim J, Kim K, Lee S: Clinical validity of the lung cancer biomarkers identified by bioinformatics analysis of public expression data. Cancer Res 2007, 67:7431-8.

21. Schwarte-Waldhoff I, Klein S, Blass-Kampmann S, Hintelmann A, Eilert C, Dreschers S, Kalthoff H, Hahn SA, Schmiegel W: DPC4/SMAD4 mediated tumor suppression of colon carcinoma cells is associated with reduced urokinase expression. Oncogene 1999, 18:3152-3158. 
22. Muller N, Reinacher-Schick A, Baldus S, van Hengel J, Berx G, Baar A, van Roy F, Schmiegel W, Schwarte-Waldhoff I: Smad4 induces the tumor suppressor E-cadherin and P-cadherin in colon carcinoma cells. Oncogene 2002, 21:6049-6058.

23. Caswell PT, Spence HJ, Parsons M, White DP, Clark K, Cheng KW, Mills GB, Humphries MJ, Messent AJ, Anderson KI, McCaffrey MW, Ozanne BW, Norman JC: Rab25 associates with alpha5beta1 integrin to promote invasive migration in 3D microenvironments. Dev Cell 2007, 13:496-510.

24. Singer $S$, Malz M, Herpel E, Warth A, Bissinger M, Keith M, Muley T, Meister M, Hoffmann H, Penzel R, Gdynia G, Ehemann V, Schnabel PA, Kuner R, Huber P, Schirmacher P, Breuhahn K: Coordinated expression of stathmin family members by far upstream sequence element-binding protein-1 increases motility in non-small cell lung cancer. Cancer Res 2009, 69:2234-2243.

25. Basu GD, Azorsa DO, Kiefer JA, Rojas AM, Tuzmen S, Barrett MT, Trent JM, Kallioniemi O, Mousses S: Functional evidence implicating S100P in prostate cancer progression. Int J Cancer 2008, 123:330-339.

26. Mangala LS, Arun B, Sahin AA, Mehta K: Tissue transglutaminase-induced alterations in extracellular matrix inhibit tumor invasion. Mol Cancer 2005, 4:33.

27. Ai L, Kim WJ, Demircan B, Dyer LM, Bray KJ, Skehan RR, Massoll NA Brown KD: The transglutaminase 2 gene (TGM2), a potential molecular marker for chemotherapeutic drug sensitivity, is epigenetically silenced in breast cancer. Carcinogenesis 2008, 29:510-518.

28. Nikolova DA, Asangani IA, Nelson LD, Hughes DP, Siwak DR, Mills GB, Harms A, Buchholz E, Pilz LR, Manegold C, Allgayer H: Cetuximab attenuates metastasis and $\mathrm{u}$-PAR expression in non-small cell lung cancer: U-PAR and E-cadherin are novel biomarkers of cetuximab sensitivity. Cancer Res 2009, 69:2461-2470

29. Rana S, Maples PB, Senzer N, Nemunaitis J: Stathmin 1: a novel therapeutic target for anticancer activity. Expert Rev Anticancer Ther 2008, 8:1461-1470

30. Bartling B, Rehbein G, Schmitt WD, Hofmann HS, Silber RE, Simm A: S100A2-S100P expression profile and diagnosis of non-small cell lung carcinoma: impairment by advanced tumour stages and neoadjuvant chemotherapy. Eur J Cancer 2007, 43:1935-1943.

31. Diederichs S, Bulk E, Steffen B, Ji P, Tickenbrock L, Lang K, Zänker KS, Metzger R, Schneider PM, Gerke V, Thomas M, Berdel WE, Serve H, MüllerTidow C: S100 family members and trypsinogens are predictors of distant metastasis and survival in early-stage non-small cell lung cancer. Cancer Res 2004, 64:5564-5569.

32. Offersen BV, Pfeiffer $P$, Andreasen P, Overgaard J: Urokinase plasminogen activator and plasminogen activator inhibitor type-1 in nonsmall-cell lung cancer: relation to prognosis and angiogenesis. Lung Cancer 2007, 56:43-50.

33. Watanabe T, Tanaka G, Hamada S, Namiki C, Suzuki T, Nakajima M, Furihata C: Dose-dependent alterations in gene expression in mouse liver induced by diethylnitrosamine and ethylnitrosourea and determined by quantitative real-time PCR. Mutat Res 2009, 673:9-20.

doi:10.1186/1475-2867-10-2

Cite this article as: Watanabe et al:: Comparison of lung cancer cell lines representing four histopathological subtypes with gene expression profiling using quantitative real-time PCR. Cancer Cell International 2010 10:2.

\section{Publish with Bio Med Central and every scientist can read your work free of charge}

"BioMed Central will be the most significant development for disseminating the results of biomedical research in our lifetime. "

Sir Paul Nurse, Cancer Research UK

Your research papers will be:

- available free of charge to the entire biomedical community

- peer reviewed and published immediately upon acceptance

- cited in PubMed and archived on PubMed Central

- yours - you keep the copyright 